\title{
Temperature measurement on neurological pulse generators during MR scans
}

\author{
Wolfgang Kainz*1, Georg Neubauer ${ }^{1}$, Richard Überbacher ${ }^{1}$, François Alesch ${ }^{2}$ \\ and Dulciana Dias Chan ${ }^{3}$
}

Address: ${ }^{1}$ Department of Mobile Communications Safety, ARC Seibersdorf Research, Austria, ${ }^{2}$ Department of Neurosurgery, AKH Vienna, Austria and ${ }^{3}$ Department of Biomedical Engineering, Johns Hopkins University, MD, USA

E-mail: Wolfgang Kainz* - kainz@gmx.com; Georg Neubauer - georg.neubauer@arcs.ac.at;

Richard Überbacher - richard.ueberbacher@arcs.ac.at; François Alesch - francois.alesch@univie.ac.at; Dulciana Chan - dulciana@jhu.edu

${ }^{*}$ Corresponding author

Published: 12 September 2002

BioMedical Engineering OnLine 2002, 1:2

This article is available from: http://www.biomedical-engineering-online.com/content/l///2

(C) 2002 Kainz et al; licensee BioMed Central Ltd. This article is published in Open Access: verbatim copying and redistribution of this article are permitted in all media for any non-commercial purpose, provided this notice is preserved along with the article's original URL.
Received: 10 July 2002

Accepted: 12 September 2002

\begin{abstract}
According to manufacturers of both magnetic resonance imaging (MRI) machines, and implantable neurological pulse generators (IPGs), MRI is contraindicated for patients with IPGs. A major argument for this restriction is the risk to induce heat in the leads due to the electromagnetic field, which could be dangerous for the surrounding brain parenchyma. The temperature change on the surface of the case of an ITREL-III (Medtronic Inc., Minneapolis, MN) and the lead tip during MRI was determined. An anatomical realistic and a cubic phantom, filled with phantom material mimicking human tissue, and a typical lead configuration were used to imitate a patient who carries an IPG for deep brain stimulation. The measurements were performed in a I.5 T and a 3.0 T MRI. $2.1^{\circ} \mathrm{C}$ temperature increases at the lead tip uncovered the lead tip as the most critical part concerning heating problems in IPGs. Temperature increases in other locations were low compared to the one at the lead tip. The measured temperature increase of $2.1^{\circ} \mathrm{C}$ can not be considered as harmful to the patient. Comparison with the results of other studies revealed the avoidance of loops as a practical method to reduce heating during MRI procedures.
\end{abstract}

\section{Introduction}

Magnet resonance imaging (MRI) is a very powerful diagnostic tool in clinical neuroscience and hence well established in neuroimaging. Implanted electronic devices like neurological pulse generators used for instance in the treatment of chronic pain or advanced Parkinson's disease are commonly considered to cause a contraindication for the use of MRI. Neurological pulse generators are typically used in the treatment of Parkinson's disease. Located in the chest region under the skin they stimulate certain areas of the brain, namely the thalamus or subthalamus $[1,2]$. Due to its superior programming possibilities, our center prefers the IPG type ITREL-III and ITREL-II. A programming device can telemetrically adjust the stimulation parameters such as frequency, amplitude and pulse duration. The major reason for this contraindication is the likelihood to cause uncontrolled temperature elevations by current induction due to the electromagnetic field of the MRI. In many cases this diagnostic restriction may be compensated by the use of other neuro-imaging facilities like computer tomography. But there are cases where MRI is mandatory. However, closing this important diagnostic window for the carrier of neurological IPGs remains a serious problem. On the other hand, we do not have clear 
evidence that there are significant and critical temperature elevations induced by MRI. Few investigations are available in both medical and technical literature. Moreover, these are contradictory.

Gleason et al. [3] investigated several IPGs, including the ITREL-II (Medtronic Inc., Minneapolis, MN) neurostimulator in 0.35 Tesla (T) and 1.5 T MR Systems. They found a temperature increase on the implant surface of $4.2^{\circ} \mathrm{C}$ during a 12 minute scan in the $1.5 \mathrm{~T}$ MR system using the radio frequency (RF) body coil. The temperature measurement was conducted in air (without phantom material) with the fiber optic temperature measurement system Luxtron 790 . Neither a realistic phantom nor a phantom liquid was used. The positioning of the implant, the leads and the electrodes were not reported. Within the study of Gleason et al. the used imaging parameters are not described, therefore it is not possible to compare these settings with those applied in the frame of the presented work. It should be noted that investigations performed by Gleason et al. were performed using a 0.35 and $1.5 \mathrm{~T} \mathrm{MRI}$, while we performed the tests in a 1.5 and a 3 T MRI. However, Gleason et al. performed their investigations in air, without using a tissue simulating liquid. This was a major difference in the study design. Gleason et al. concluded in their study that the temperature rise measured in air can be considered as a worst case condition, since the heating effect of a device that is implanted would be reduced by the heat conduction of the surrounding tissue. Apart from that, Gleason et al. found the maximum temperature increase on the case of the investigated pulse generator. In the frame of our study, the maximum temperature increase was found at the tip of the electrodes. The fact that the imaging parameters used within the study of Gleason et al. were not reported, that the maximum temperature found was not at the same location on the implant and, most importantly, that no phantom was used in the study of Gleason et al. makes comparisons between the results of both studies not very meaningful.

Tronnier et al. [4] used a sphere phantom filled with a salt-water solution and studied heating effects for the ITREL-II and ITREL-III (Medtronic Inc., Minneapolis, $\mathrm{MN}$ ) in a $0.2 \mathrm{~T}, 0.25 \mathrm{~T}$ and $1.5 \mathrm{~T}$ MRI using RF head and body coils. The temperature was measured using an infrared camera. A maximum increase below $0.15^{\circ} \mathrm{C}$ on the implant and the leads was observed. However, it is questionable to use an infra-red camera to measure the temperature of parts of electronic implants inside a phantom [6]. A temperature increase observed using an infrared camera would not show the maximum temperature increase on the implant situated in a phantom or a body, but would only show the temperature increase on the surface of the body. Therefore this type of measurement pro- cedure was not adequate to find temperature increase inside the body.

Rezai et al. $[5,6]$ found temperature changes from $2 \cdot 5^{\circ} \mathrm{C}-$ $25.3^{\circ} \mathrm{C}$ in the leads or the can of the deep brain stimulator Soletra 7426 (Medtronic Inc., Minneapolis, MN) using the body RF coil of a $1.5 \mathrm{~T} / 64 \mathrm{MHz}$ MRI system depending on the SAR level used and how the lead wires were positioned. Using the head RF coil, the temperature change ranged from $2.3^{\circ} \mathrm{C}-7.1^{\circ} \mathrm{C}$. They concluded that excessive heating occured under certain ("worst case") conditions but that MRI-related heating did not appear to present a major safety concern for patients with the bilateral neurostimulation system Soletra 7426 (Medtronic Inc., Minneapolis, $\mathrm{MN}$ ), as long as guidelines pertaining to the position of these devices and MRI parameters were carefully adhered to. They specified a list of 11 safety guidelines.

There are several advantages in using MRI for patients with neurostimulators, however information on MRI safety aspects are scarce. At the time these investigations were performed, only two studies on this specific topic were available to us. Gleason et al. examined heating effects on the implants surface without using a phantom representing the human body. Their results showed that considerable heating on the implant and the leads could occur, however, these results were not adequate to draw any conclusions on the temperature increase on IPGs in humans examined in MRIs. Tronnier et al. used infrared cameras to monitor temperature increase of IPGs situated in a phantom. Therefore no information on the highest temperature increase close to the implant or the lead was obtained. We decided to perform these investigations to obtain information on the temperature increase in MRIs in the vicinity of NPGs and their electrodes under realistic conditions. The aim of the present study was to determine the temperature change on the surface of the case of an ITREL-III (Medtronic Inc., Minneapolis, MN) and the lead tip during MRI.

\section{Methods}

The temperature measurements were performed in vitro using a phantom especially developed for examinations with the ITREL-III (Medtronic Inc., Minneapolis, MN). The examinations were carried out on one implant, with standard electrodes (Medtronic Type \# 3387) and leads with a length of 51 centimeter $(\mathrm{cm})$. The neurostimulator was placed in the upper left quadrant of the thorax portion of the phantom. The placement was within $1 \mathrm{~cm}$ of the phantom surface. During the measurements the ITREL-III was set to the "off" mode (i.e. no stimulation output). The extensions were connected to the neurostimulator and the excess lengths were wrapped once around the implant case. The lead was positioned with a small 


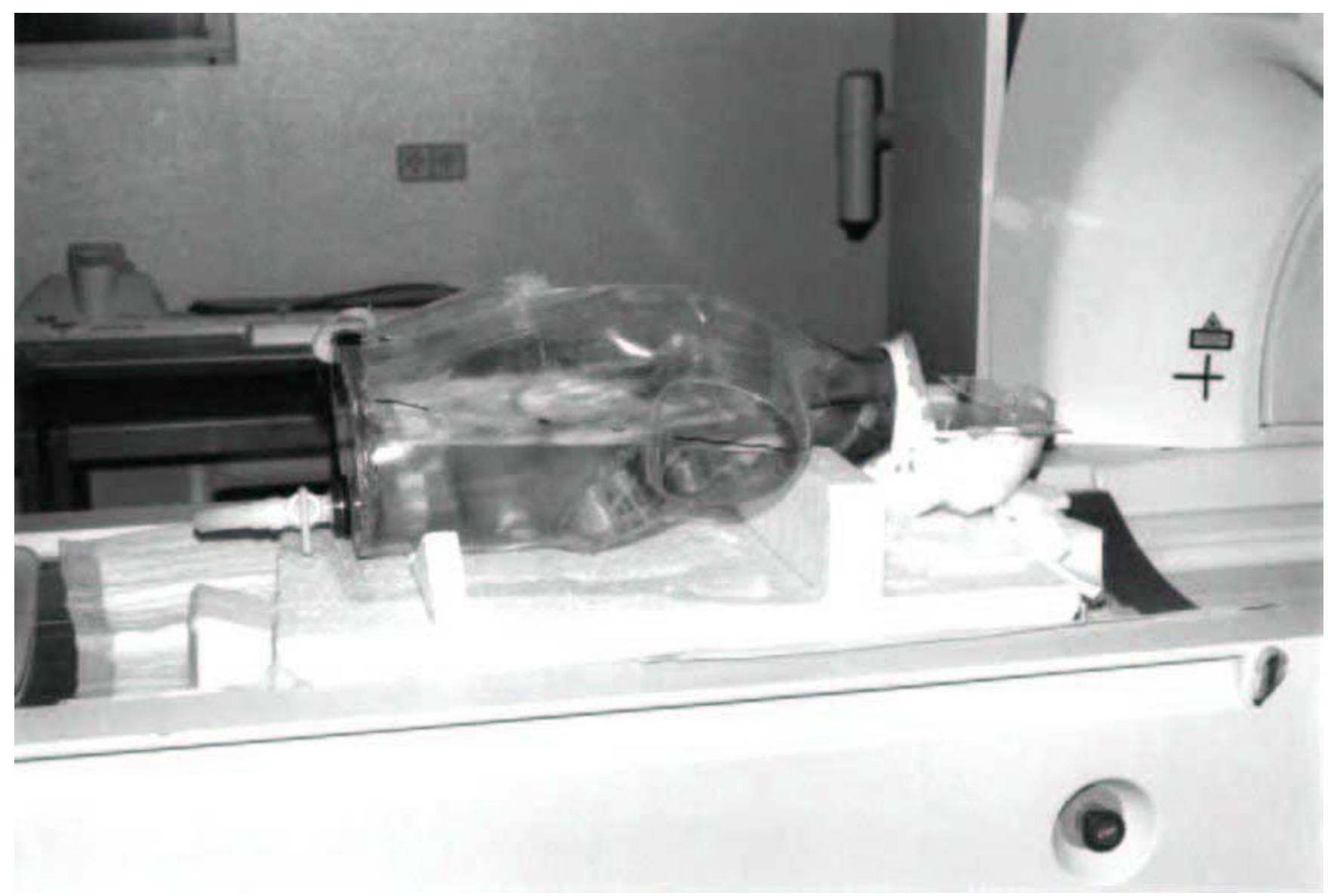

Figure I

Phantom used in the I.5 T MRI

Table I: Ingredients of the phantom material in mass \%

\begin{tabular}{ccc}
\hline Material & \multicolumn{2}{c}{ Phantom Liquid } \\
\cline { 2 - 3 } & Brain & Muscle \\
\cline { 2 - 3 } & {$[\%]$} & {$[\%]$} \\
Water & & \\
Ethylene Glycol & 52.54 & 63.94 \\
Natrium Chloride & 46.52 & 35.11 \\
& 0.94 & 0.95 \\
\hline
\end{tabular}

single loop (3.5 $\mathrm{cm}$ in diameter) at the skull and $7 \mathrm{~cm}$ deep into the head perpendicular to the surface of the skull. This positioning corresponds to a typical placement of the ITREL-III in deep brain stimulation. The phantom consisted of three parts: the skull, the trunk and the skull- trunk connecting tube (see Fig. 1). The skull-trunk connection tube of the phantom established the electrical connection between the two liquid phantom materials (brain and muscle liquid) without mixing the liquids. The inside diameter of the connection tube measured $44 \mathrm{~mm}$ and was filled with a jelly phantom material imitating muscle tissue. The skull and trunk was filled with phantom materials mimicking brain and muscle tissue. The ingredients of the phantom materials are listed in Table 1. Temperature measurements in a radio frequency environment require materials that have the following characteristics: correct dielectric properties and correct specific thermal constant. New phantom materials [9] were developed for brain and muscle tissue. They were liquid and mixed according to the dielectric properties given for 63.6 $\mathrm{MHz}$. This frequency corresponded to the frequency of the frequently used 1.5 T MRI. The parameters of the phantom liquids (the permittivity, the conductivity and the specific thermal constant) were all within an acceptable range. The dielectric properties of human tissue were taken from [7]. Properties of the phantom liquids were measured with the "Dielectric Probe Measurement System" HP 
Table 2: Dielectric (permittivity $\in$ ' and the conductivity $\in$ ") and thermal properties (specific heat $c$ ) of human tissue and phantom materials for $63.6 \mathrm{MHz}$

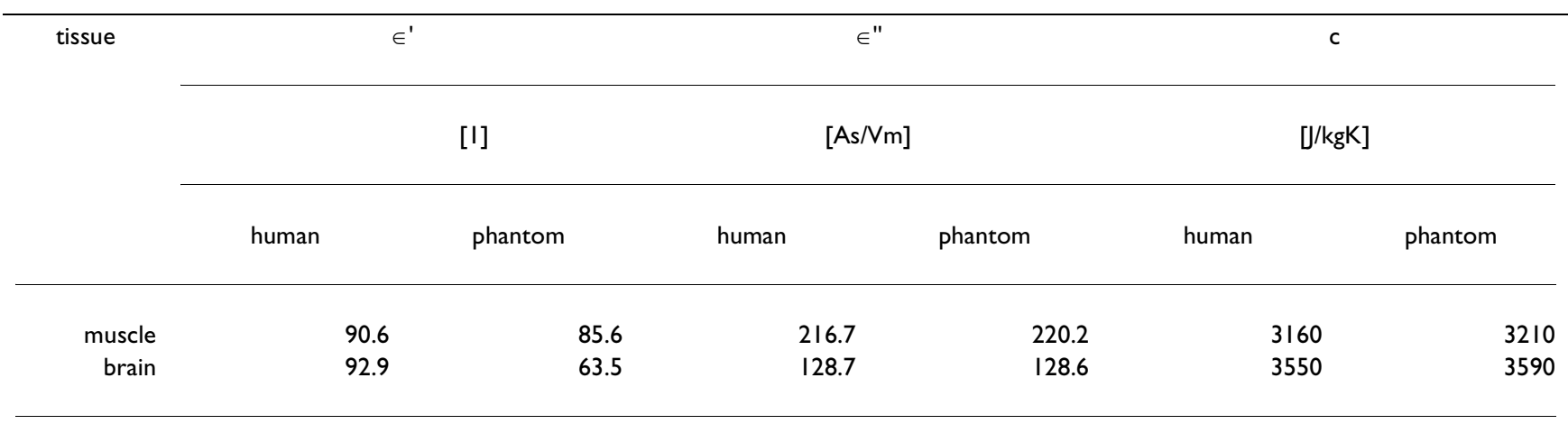

Table 3: Parameters of the turbo spin echo sequence used during the temperature measurements

\begin{tabular}{lrr}
\hline \multicolumn{1}{c}{ MRI - Parameter } & I.5 T MRI & 3.0 T MRI \\
\cline { 2 - 3 } & TSE & \\
\hline & & TSE \\
TR [msec] & & 1300 \\
TE [msec] & 600 & 20 \\
Thickness [mm] & 18 & 4 \\
Slices & 6 & 13 \\
Number of acquisitions & 17 & 14 \\
Matrix [elements] & 14 & $252 \times 256$ \\
Field of view [mm] & 400 cube & 256 \\
Duration [sec] & 707 & 70 cube \\
& & \\
\end{tabular}

85070 from Hewlett Packard. The thermal constant was measured by a method described in [8]. Table 2. summarizes the dielectric properties of human tissue and phantom materials.

The temperature was measured by the fiber optic temperature measurement system Luxtron 790. This system included four temperature probes allowing simultaneous measurement at four different locations. Two of them were used for temperature measurement on the implant surface, the third for measurement of the air temperature in the MRI tube and the fourth for the lead tip. The Luxtron 790 achieved a resolution better than $0.1^{\circ} \mathrm{C}$. For correct temperature measurements it was necessary to ensure optimal contact of the fiber optic temperature probes with the measured surface. This was achieved by arranging all four probes in the shape of an arc, which pressed the probe on the lead tip or the implant case. Thermal balance was considered a critical and important factor. All temper- ature measurements are overlaid by a heating or cooling process which leads to distorted results. This would occur if the phantom liquid and the implant were not thermally balanced (e.g. due to air conditioning in the MRI room). Thus, 24 hours before measurements, the phantom with implant and the temperature measurement system were put into the MRI room. For all measurements, the MRI parameters were set to values usually used for brain MR imaging which resulted in a maximum local specific absorption rate (whole body SAR) of $2 \mathrm{~W} / \mathrm{kg}$, estimated by the SAR monitor of the MR machine. Table 3 summarizes the parameters used for the MRI procedure.

\section{Measurements at I.5 T}

The measurements were performed in a $1.5 \mathrm{~T}$ MR Imager from Siemens (Magnetom) working at $63.6 \mathrm{MHz}$. The temperature was measured at four positions: at two points at the surface of the implant (see Fig. 2: P2 and P3), at the surface of the lead-tip (electrode pole \# 3) and in air $5 \mathrm{~cm}$ 


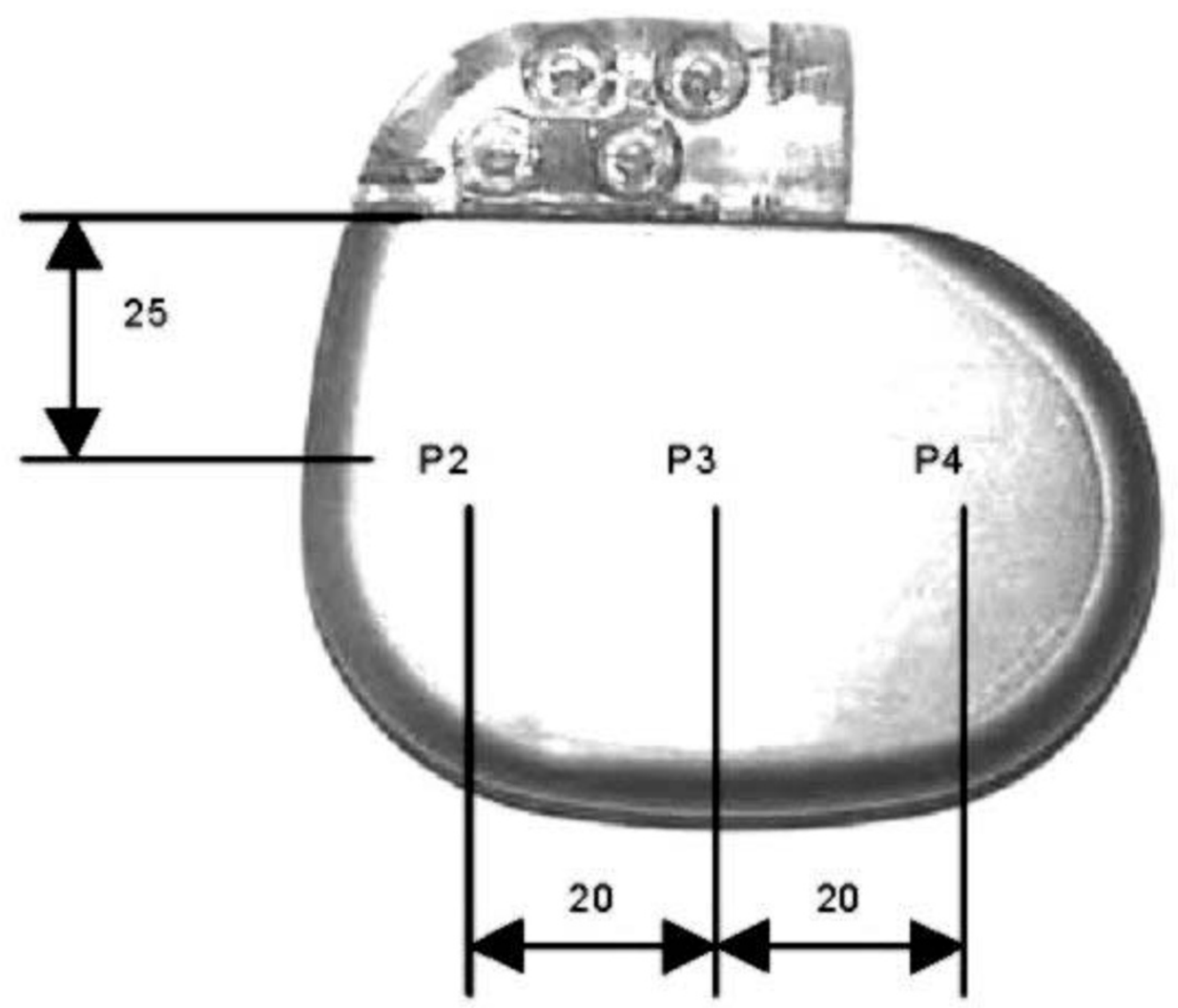

Figure 2

Measurement positions on the implant case (dimensions in $\mathrm{mm}$ )

above the back of the phantom. The air temperature was measured as a reference temperature for the measurements at the implant surface and at the lead-tip. The two experiments had two different fields of view (FOV) which were changed electronically. One FOV was situated in the head above the lead tip and the other was in the chest region directly over the implant. In both cases the TSE sequence was used (see Tab. 3).

\section{Measurements at 3.0 T}

In addition, temperature measurements in a $3.0 \mathrm{~T}$ MRI from Bruker, working at 125.6 MHz were performed. The objective of this measurement was to find out if an even higher temperature increase could be detected on the surface of the implant or the lead using this kind of MRI. Because of the shorter inner coil dimensions of the $3.0 \mathrm{~T}$ MRI, a smaller phantom was used: an acryl glass cube with a side length of $160 \mathrm{~mm}$. The cube phantom provided a similar lead configuration as the phantom described earlier, but its outer dimensions were small enough to fit into the coil. Because the lead-tip and the implant case were closer than in the realistic phantom, the FOV covered both. As in the experiment at $1.5 \mathrm{~T}$ a TSE sequence was used with a maximum local SAR of $2 \mathrm{~W} / \mathrm{kg}$ (see Tab. 3 ).

\section{Results}

Measurements at I.5 T

It could be shown that the temperature inside the MRI tube was about $1^{\circ} \mathrm{C}$ higher than the outside. This indicated a slight and slow overlay of the temperature increase. But due to slow temperature transients of the phantom liquid this temperature overlay was only delayed and had no significant contribution to any temperature increase of metallic parts of the implant within the phantom liquid. The measured temperature increase of the implant itself was a modest value. An increase of about $0.25^{\circ} \mathrm{C}$ was 


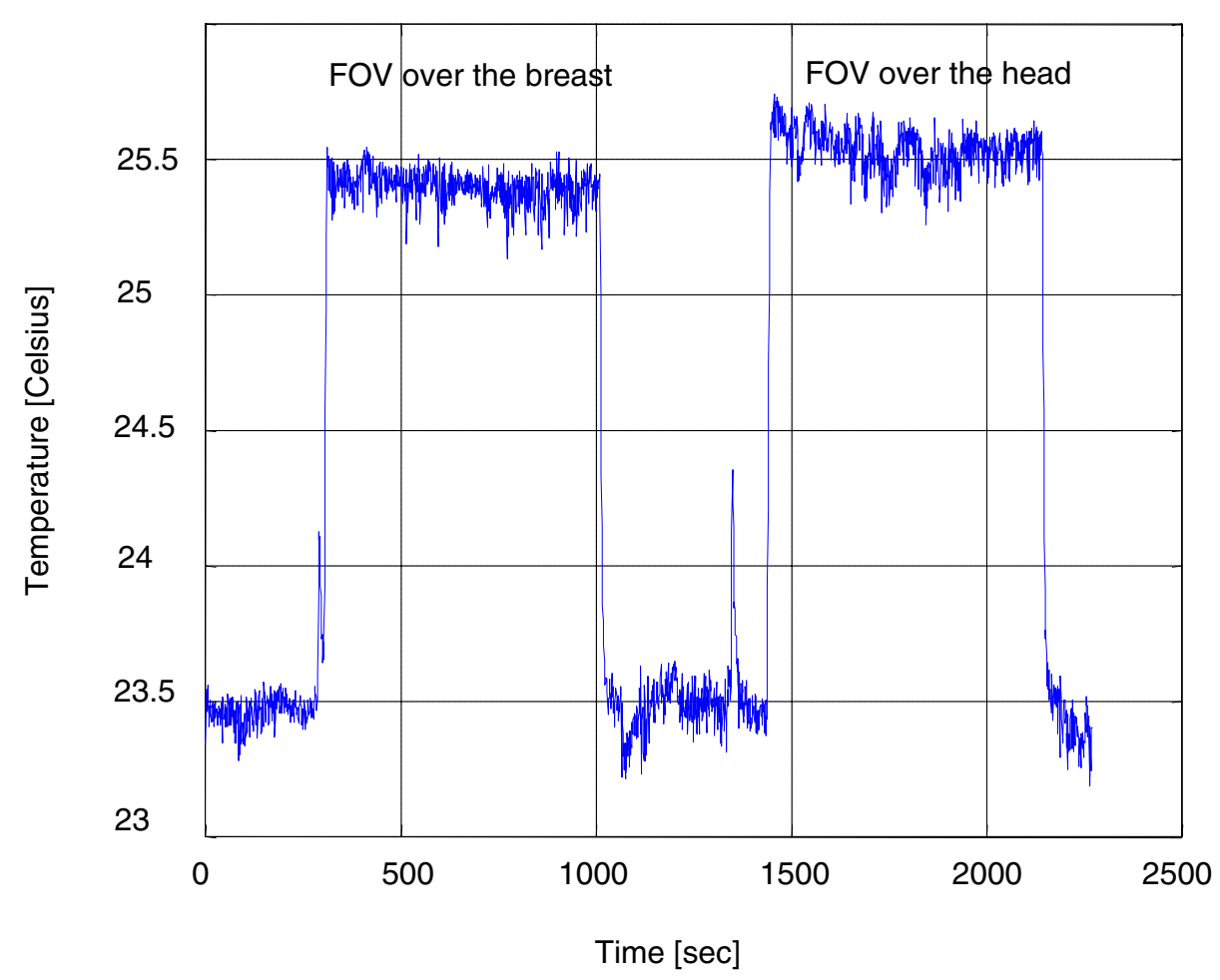

Figure 3

Temperature at the lead tip as a function of time for two different FOV's (FOV over the breast: 300 to 1007 seconds; FOV over the head: 1434 to 214 I seconds)

shown. But at the lead tip a higher temperature increase was found. If the field of view was put over the implant the temperature increased about $1.8^{\circ} \mathrm{C}$. With the FOV over the lead tip, an increase of $2.1^{\circ} \mathrm{C}$ was measured. Figure 3 shows the temperature increase versus time. Remarkable was the short increase and decrease time of about 20 seconds. This short time constant was possibly due to the small mass of the lead tip. The induced RF current dissipated in the tissue and heated the surrounding tissue and the lead tip.

\section{Measurements at 3.0 T}

The results were similar to the results at the $1.5 \mathrm{~T}$ machine: slight and slow temperature increase at the implant and fast and higher temperature increase at the lead tip. The increase of the implant itself was $0.2^{\circ} \mathrm{C}$. Almost the same was measured in the realistic phantom at $1.5 \mathrm{~T}$. At the lead tip, the temperature increased $0.8^{\circ} \mathrm{C}$. The temperature at the lead tip versus time is shown in Figure 4.

\section{Conclusion}

It was shown that the lead-tip is the most critical part in respect of heating problems of IPGs exposed in MRI. The highest measured temperature increase was $2.1^{\circ} \mathrm{C}$. An increase of such magnitude was only found at the tips of the leads. The $2.1^{\circ} \mathrm{C}$ temperature increase only occurred locally because of the small mass of the lead-tip. Switching the RF field was synchronous with the temperature increase and decrease at the lead-tip. It was a direct result of induced RF currents in the lead dissipating in the phantom liquid and heating the lead tip and the surrounding tissue. Due to the higher temperature in the MRI tube, the temperature of the phantom increased slowly. It can be assumed that the temperature increase at the lead tip in a patient would be smaller due to blood flow in the brain. Based on threshold temperatures known to produce reversible $\left(42^{\circ} \mathrm{C}-44^{\circ} \mathrm{C}\right)$ and irreversible $\left(>45^{\circ} \mathrm{C}\right)$ thermal lesions, the measured temperature increase of $2.1^{\circ} \mathrm{C}$ can not be considered as harmful to the patient. 


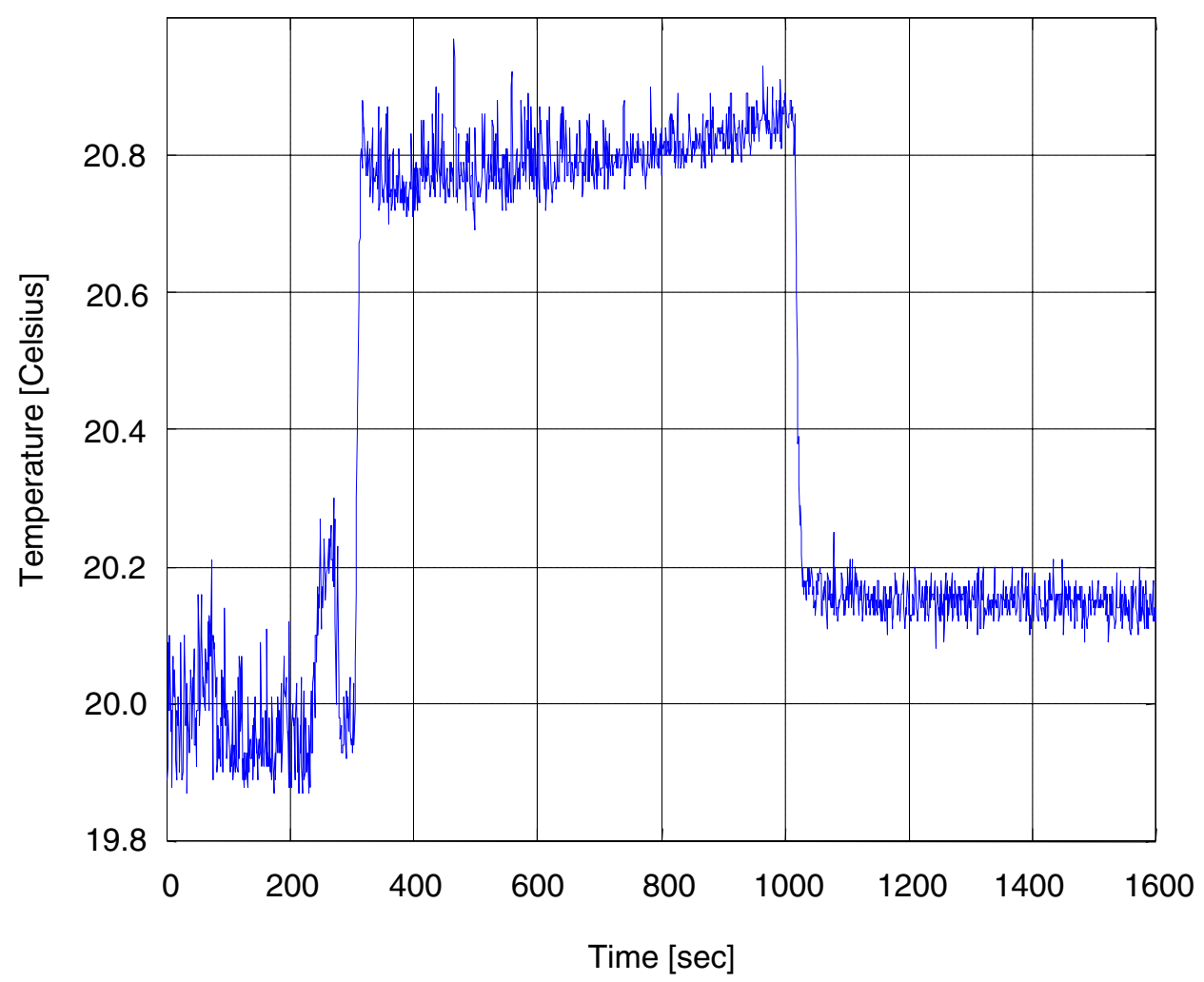

\section{Figure 4}

Temperature at the lead tip as a function of time for the measurements at the $3.0 \mathrm{~T}$ MRI (MR-scan: from 300 to I00I seconds)

The highest temperature increase observed by Rezai et al. on the leads/electrodes was $7.1^{\circ} \mathrm{C}$ (whole body SAR 3.9 $\mathrm{W} / \mathrm{kg}$ ) showing more elevated temperature increases compared to our results $\left(2.1^{\circ} \mathrm{C}\right.$, whole body SAR $\left.2 \mathrm{~W} / \mathrm{kg}\right)$. We found only small temperature increases up to $0.25^{\circ} \mathrm{C}$ on the implant itself. These results are not in agreement with the results of Rezai et al. where temperature increases up to $25^{\circ} \mathrm{C}$ were found on the implant case. However, it has to be taken into account that Rezai et al. used a bilateral neurostimulation system, while we used a unilateral system. Furthermore Rezai et al. wrapped the lead extension two or four times around the perimeter. A $25^{\circ} \mathrm{C}$ temperature increase on the implant case was found when the lead extension was wrapped four times around the perimeter of the neurostimulator. Wrapping the extended lead only two times around the perimeter reduced the temperature increase at the implant case to $6^{\circ} \mathrm{C}$. Reducing the number of loops by a factor of two reduced the temperature increase by a factor of four, which is evident since the amount of induced current in a coil is proportional to the number of loops and the temperature increase is proportional to the square of the current.

The differences between Rezai et al. and our experiments were that in our experiment the lead extension was wrapped only once around the perimeter of the implant and the whole body SAR was $2 \mathrm{~W} / \mathrm{kg}$ instead of $3.9 \mathrm{~W} / \mathrm{kg}$. In order to compare the results of Rezai et al. with ours, we scaled their findings of a $6^{\circ} \mathrm{C}$ temperature increase with two loops at $3.9 \mathrm{~W} / \mathrm{kg}$ (whole body SAR) to our experimental conditions with one loop and $2 \mathrm{~W} / \mathrm{kg}$ (whole body SAR). This theoretical temperature increase of the implant case was determined to be $0.8^{\circ} \mathrm{C}$. We measured a $0.25^{\circ} \mathrm{C}$ temperature increase on the implant case which 
could be due to different phantom materials, lead configurations and positions of the phantom in the MRI. However, this clearly indicates the avoidance of loops is necessary to reduce MRI related heating. Numerical calculations [10] have shown that a sophisticated lead configuration can reduce the induced current during RF exposure. The magnitude of induced currents can be significantly reduced through the avoidance of loops. Instead of wrapping the lead extension around the perimeter of the implant, the lead should be arranged in the form of a meander. The clinical feasibility of using a meander instead of loops has been demonstrated on several patients with implanted neurostimulators at our center.

Further investigations are necessary to find out if the proposed meander configuration is able to minimize the temperature increase for different MRIs, different imaging sequences and different lead configurations. The findings are restricted to the tested neurostimulation system ITREL-III (Medtronic Inc., Minneapolis, MN), extensions, leads, MR imager and especially the lead configuration near the implant case and the entry point into the skull.

\section{Acknowledgements}

The authors would like to thank Dr. Frans Gielen and Dr. Wolfgang Ladisich from Medtronic Inc. for their support and the supply with IPGs and the programming unit. In addition, thanks to the MR team from the AKH Vienna, especially Dr. Imhof for their help with the MRI.

\section{References}

I. Alesch F, Pinter M, Helscher R, Fertl L, Benabid A, Koos W: Stimulation of the ventral intermediate thalamic nucleus in tremor dominated Parkinson's disease and essential tremor. Acta Neurochirurg 1995, 136:75-81

2. Limousin P, Pollak P, Benazzouz A, Hoffmann D, Le Bas J, Brous-solle $E$, Perret J, Benabid A: Effect of parkinsonian signs and symptoms of bilateral subthalamic nucleus stimulation. Lancet 1995, 345:91-95

3. Gleason C, Kaula N, Hricak H, Schmidt R: The effect of magnetic resonance Imagers on implanted neurostimulators. Pacing \& Clinical Electrophysiology 1992, 15:81-94

4. Tronnier V, Staubert A, Hähnel S, Sarem-Aslani A: Magnetic resonance imaging with implanted neurostimulators. Neurosurgery 1999, 44:118-126

5. Rezai A, Finelli D, Rugieri P, Tkach J, Nyenhuis J, Shellock F: Letter to the Editor - Neurostimulators: Potential for excessive heating of deep brain stimulation electrodes during magnetic resonance imaging. Journal of Magnetic Resonance Imaging 200I, I 4:488-489

6. Rezai A, Finelli D, Nyenhuis J, Hrdlicka G, et al: Neurostimulation systems for deep brain stimulation: in vitro evaluation of magnetic resonance imaging-related heating at 1.5 tesla. Journal of Magnetic Resonance Imaging 2002, 15:24 I-250

7. Gabriel S, Lau R, Gabriel C: The dielectric properties of biological tissues: II. Measurements in the frequency range $10 \mathrm{~Hz}$ to 20 GHz. Physics in Medicine \& Biology 1996, 4I:225I-2269

8. Schmid G, Neubauer G, Garn H, Molla-Djafari H: Strahlungsabsorption im menschlichen Kopf bei Exposition in hochfrequenten elektromagnetischen Feldern. AUVA Report 1998, 19:

9. Überbacher R: Neurologische Impulsgeneratoren im hoch und nieder-frequenten elektromagnetischen Feld. Diploma Thesis Technical University Vienna 200 I

10. Kainz W: Electromagnetic Interference of Electronic Implants by Mobile Phones. Doctoral Thesis Technical University Vienna 2000

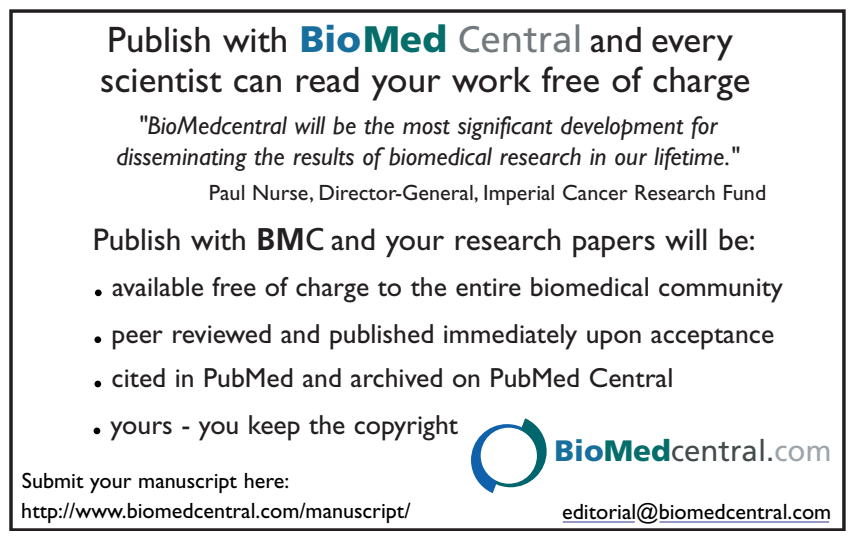

